\title{
Una sociología organizativa de la racionalización de las políticas económicas en España y Dinamarca en la era de la globalización neoliberal
}

\author{
An organizational sociology of rationalization of economic \\ policies in Spain and Denmark in the age of neoliberal \\ globalization.
}

Manu Ahedo Santisteban • mahe@soc.ku.dk

UNIVERSIDAD DE COPENHAGUE, DEPARTAMENTO DE SOCIOLOGÍA, DINAMARCA

Recibido: $19 / 12 / 2017$

Aceptado: $16 / 10 / 2018$

\section{Resumen}

En la era de globalización económica neoliberal el proceso de racionalización, como instrumento de decisión y mito normativo, ha intensificado su tecnocratización. Esto es evidente en los ámbitos más complejos de la sociedad como la economía. Sin embargo, una política económica democrática debe mantener un difícil y virtuoso equilibrio entre la racionalidad político-democrática y la racionalidad técnico-científica, dentro de unas precondiciones locales en el funcionamiento de la democracia representativa y de la ciencia económica. El papel de economistas académicos y profesionales en la política económica es un aspecto adecuado para analizar la tensión entre las dos racionalidades. Con datos de fuentes digitales oficiales se compara la participación de economistas en la política económica en tres contextos organizativos: a) como altos técnicos en la administración pública; b) en gobiernos y ministerios; y c) en órganos públicos de asesoramiento y regulación económica. La comparación muestra realidades y tipos diferentes. En Dinamarca se observa un tipo democrático-académico: dentro del control del parlamento los economistas gozan de una relativamente alta y transparente autonomía como académicos asesores y tecnócratas públicos. En España se observa un tipo político-tecnocrático: dentro del amplio poder del gobierno ejecutivo los economistas tienen un relativamente bajo nivel de autonomía y los tecnócratas públicos tienen un alto control político. Estas diferencias derivan de las particulares trayectorias institucional y organizativa del estado en los dos países. Algunos efectos de estas diferencias institucionales en la política económica son observables en el diferente funcionamiento general y sectorial de las dos economías nacionales.

Palabras clave: democracia, tecnocracia, expertos, administración pública, organizaciones tecno-racionalizadas.

\section{Abstract}

In the age of neoliberal economic globalization, rationalization process, as cognitive instrument and as normative myth, has intensified its tendency towards technocrazitation. This is more evident in society's most complex domains, such as the economy. It is assumed, however, that an economic policy in a democratic society requires a difficult and virtuous balance between politico-democratic rationality and technical-scientific rationality, within local preconditions related to the working of representative democracy and economic sciences. The role of academic and professional economists in economic policy is an adequate domain to analyse the tension between these two rationalities. With data from official digital sources, the policy participation of economists in three organizational contexts is compared: a) as high-level technocrats in public administration; b) as members of government or ministries; and c) as members of advisory and regulatory public organizations. The rationalization of economic policy in the two countries displays two different realities and types. 
In Denmark a democratic-scientific model is observed: within parliament's control economists have a relatively high level of autonomy and transparency, both as academic advisors and as public technocrats. In Spain a political-technocratic model is found: within executive government's ample power economists have a relatively low level of autonomy and public economic technocrats are under political control. These differences derive from state's particular institutional and organizational trajectories in the two countries. Some of the effects of these institutional differences in economic policymaking can be observed in the different general and sectorial performance of the two national economies.

Keywords: democracy, technocracy, experts, public administration, techno-rationalized organizations.

\section{INTRODUCCIÓN}

En la era de la globalización económica neoliberal la gestión política de la economía es uno de los retos más difíciles para los gobiernos democráticos (Rodrik, 2011). Deben hacer frente a dos tipos de poderes. Primero, las políticas económicas son crecientemente percibidas como políticas públicas con una alta complejidad técnica, y el poder y la influencia de la ciencia económica en las políticas económicas se ha convertido en una cuestión relevante y crítica (Hirschman y Berman, 2014). Segundo, el poder del capital y de las grandes empresas (poder estructural, poder instrumental y poder ideacional o discursivo), y su capacidad de influencia sobre las políticas económicas (Beck, 2004; Woll, 2016). Las políticas económicas en una sociedad democrática manifiestan una clara tensión entre la lógica político-democrática y la lógica técnico-científica. En general, la debilidad de las democracias ante estos poderes, las recurrentes crisis financieras y escándalos fiscales, y la persistente desigualdad socioeconómica, explican parte de la creciente incertidumbre sociopolítica.

La política económica es un caso de racionalización técnica y organizativa de las sociedades contemporáneas. La lógica y racionalidad técnica dentro del sistema de poder y decisional del estado es un elemento clave en las democracias liberales. En las últimas décadas el proceso ha aumentado su carácter técnico, y en algunos ámbitos se ha pasado de una simple lógica tecnocrática a lógicas cercanas a la expertocracia o cienciocracia. Sin embargo, se asume que para un adecuado funcionamiento de la democracia moderna se requiere un virtuoso equilibrio entre tecnocracia y democracia, basado en dos precondiciones locales: a) la tecnocracia económica depende de que la ciencia económica académica y aplicada sea claramente empírica, éticamente responsable y orientada a los problemas reales de la sociedad; y b) la democracia depende de una sana cultura democrática, basada en adecuadas regulaciones y prácticas en aspectos como la financiación de los partidos políticos, la división de poderes, la calidad del debate público, etc. ${ }^{1}$

Además del papel de economistas individuales en los gobiernos es necesario analizar la dimensión organizativa en la influencia de los economistas académicos y profesionales en

\footnotetext{
1 Ahedo (2018) realiza una comparación de algunos aspectos relativos a estas precondiciones.
} 
las políticas económicas. Su influencia a través de contextos organizativos tiene un carácter estructural y sistémico. Las organizaciones son una de las formaciones sociales donde la racionalidad técnica ha adquirido una mayor importancia (Adler, 2009). En el ámbito público o estatal el desarrollo de la racionalidad abstracta y formal ha ido dando paso a un mayor nivel de racionalización técnica. Este carácter técnico de la organización pública funciona de forma ambivalente como práctica o aspiración cognitiva sustantiva, y como fuente de legitimidad, es decir, como mito legitimador (Meyer y Scott, 1992). Dentro del estado destacan las llamadas agencias reguladoras independientes (ARI), creadas en las últimas décadas en paralelo al desarrollo del estado regulador. En el ámbito económico, las ARI incluyen desde el banco central hasta las agencias que regulan los mercados bursátiles, la competencia empresarial, y sectores relevantes como el energético, el bancario, etc. Las ARI funcionan inspiradas por la racionalidad técnica, y son un claro ejemplo de organizaciones tecno-racionalizadas. A pesar de la creciente similitud en la estructuración organizativa de los estados y en la difusión de las ARI (Polillo y Guillén, 2005; Gilardi, 2009) las diferencias entre países siguen siendo relevantes (Christensen y Lægreid, 2006; Painter y Peters, 2010).

La comparación entre España y Dinamarca sigue la estrategia de comparación de casos altamente diferentes dentro del contexto europeo. La investigación se fundamenta en diversos datos e informaciones secundarias, principalmente de fuentes digitales oficiales. Alguna información digital puede ser considerada ya como una adecuada fuente de datos para la investigación social (Marres, 2017). España es uno de los países de la zona Euro más afectados por la crisis económica de 2008, sus políticas económicas han sido objeto de vigilancia por la troika (Comisión Europea, Banco Central Europeo y Fondo Monetario Internacional), y tiene una corta tradición democrática, problemas de calidad institucional, inestabilidad macroeconómica, y una tendencia a un alto nivel de desempleo y de desigualdades socioeconómicas. Dinamarca no pertenece a la zona Euro, ya que fue rechazado en referéndum en el 2000, y tiene una larga tradición democrática, una desarrollada calidad institucional, estabilidad macroeconómica, y una tendencia a un bajo nivel de desempleo y de desigualdades socioeconómicas. ${ }^{2}$

El artículo se estructura de la siguiente manera. En el segundo apartado se presenta el marco teórico y analítico sobre la racionalización y la política económica, y sobre las organizaciones tecno-racionalizadas; y se proponen tres formas principales de influencia de los economistas en la política económica democrática: a) como altos cargos de la administración pública; b) como ministro-a o alto cargo de gobierno; c) como miembros de consejos asesores y de ARI. En el tercer apartado se comparan estas tres formas de participación de los economistas en las políticas económicas de España y Dinamarca en los últimos años. En el último apartado se sintetiza el análisis comparado a modo de conclusiones.

\footnotetext{
2 Ahedo (2017) realiza una sociología comparada e histórica entre Dinamarca y España.
} 


\section{RACIONALIZACIÓN Y POLÍTICA ECONÓMICA: LA ORGANIZACIÓN DE LA INFLUENCIA DE LOS ECONOMISTAS}

Sobre la influencia de los economistas en las políticas económicas Hirschman y Berman (2014) identifican tres factores o dimensiones principales: la autoridad profesional de los economistas; la posición institucional de los economistas en el gobierno, y el papel de la ciencia económica en la infraestructura cognitiva de la política pública. A continuación, estas dimensiones son contextualizadas y reelaboradas, y se enfatiza la participación organizativa de los economistas profesionales y académicos en la racionalización de la política económica.

\subsection{Economía, democracia y tecnocracia}

La influencia de la ciencia económica en las políticas económicas puede contextualizarse respecto a la relación entre democracia y tecnocracia (Centeno, 1993). La tecnocracia pública ha aumentado en estatus y función en las instituciones de la modernidad. ${ }^{3}$ Este auge de la tecnocracia y la expertocracia es un reto para la democracia y para las políticas públicas (Fischer, 2009). Hay dos aspectos a destacar. Primero, el creciente papel de las ideas tecnocráticas y científicas. Para Campbell (1998), en base a lo observado en los Estados Unidos de América (EUA), las ideas científicas, académicas o de expertos operan como un marcobase fundamental de segundo orden, a través de asunciones que constriñen el abanico o espectro cognitivo de posibles soluciones disponibles para los decisores políticos, y que tienen un mayor o menor grado de institucionalización en universidades punteras, think tanks y asociaciones profesionales. Segundo, el creciente papel de los expertos. En el debate sobre los expertos en las sociedades actuales hay tres posturas: frente al elogio de la autoridad experta del expertismo el constructivismo crítico cuestiona la autoridad epistémica de los expertos, y en medio autores como Brint (1994) y Collins y Evans (2002) optan por una vía intermedia que valora potencialmente el nivel de conocimiento formal y tácito de los expertos. Incluso en el caso crítico de los pronósticos macroeconómicos, donde los economistas suelen fallar y contradecirse, Collins y Evans argumentan que no cabe negarles su conocimiento tácito. Según esta perspectiva, una falta de un mínimo de reconocimiento de la autoridad experta podría ser una peligrosa vulgarización de la ciencia. Entre el expertismo acrítico y el constructivismo crítico esta tercera posición abre un adecuado espacio para analizar y comparar el funcionamiento de los expertos en las sociedades actuales.

Según las recientes teorías de sistemas sociales modernos (principalmente Luhmann, 1995), la sociedad moderna se desarrolla en base a subsistemas crecientemente complejos, diferenciados funcionalmente y con autopoiesis, con diferenciados lenguajes, códigos, teorías e interpretaciones técnicas, y medios especializados de información y comunicación. La

\footnotetext{
${ }^{3}$ El rico debate en torno a las teorías y propuestas de J. Habermas respecto a la esfera pública, la democracia y la racionalidad técnica es un ejemplo de la relevancia del tema (Kelly, 2004).
} 
economía de mercado es uno de los subsistemas más diferenciados funcionalmente, y la ciencia económica es el marco epistémico-lingüístico central de su interpretación. La diferenciación sistémica funcional genera sus propios riesgos sistémicos e inter-sistémicos. La crisis del 2008 se debe en parte a la alta diferenciación sistémica de la economía de mercado y de las políticas económicas. En las últimas décadas, la teoría económica neoclásica o neoliberal han dominado la disciplina, y han promovido una profesión con una fuerte orientación global (Fourcade, 2006). El reciente auge de las ideas neo-keynesianas como reacción a la crisis del 2008 no parece haber debilitado el paradigma epistémico neoliberal.

Ante una mayor tecnocracia y plutocracia, los gobiernos y parlamentos elegidos por la ciudadanía se enfrentan a un difícil dilema. Por un lado, se encuentran en una situación de debilidad epistémica para interpretar y discutir la compleja economía de mercado, y de debilidad estructural ante el poder del capital y las grandes empresas. Por otro lado, como responsables de la política económica tienen, en función de sus ideologías socioeconómicas, la posibilidad de contrarrestar los riesgos derivados de una excesiva diferenciación funcional del sistema económico y del creciente poder del capital.

\subsection{Una sociología comparada y organizativa}

El estado moderno es una institución fundamental que en su construcción histórica en paralelo a los estados-nación modernos ha adquirido una relevante particularidad nacional (Bourdieu, 2014). Los estudios comparados suelen comparar países con un mínimo de comparabilidad institucional. Fourcade (2009) se centra en los factores institucionales, organizativos y políticos que condicionan la evolución de disciplina de la ciencia económica en EUA, Francia y Reino Unido, y enfatiza la importancia del «orden administrativo». Pedersen y Campbell (2014) analizan los regímenes de conocimiento económico aplicado a las políticas en Alemania, Dinamarca, EUA y Francia, compuestos por el conjunto de organizaciones que producen conocimiento orientado a políticas económicas, desde think-tanks privados hasta diversas organizaciones públicas o semipúblicas, y observan que los economistas tecnócratas del estado tienen diferentes roles y estatus. En la difusión de ideas, Ban (2016) investiga la recepción de las teorías neoclásicas angloamericanas en España y Rumanía, y para el caso de España encuentra que las ideas de la economía neoclásica ordoliberal fueron adoptadas en los años 70 y 80 y adquirieron una posición dominante en los años 80 y 90; destacando el papel del llamado «círculo de Rojo», en torno a A. Rojo, director y gobernador del Banco de España, y creador y promotor del Servicio de Estudios del banco.

Los estudios comparados y de difusión cuantitativos con un número alto de países y de ARI ofrecen información relevante sobre las tendencias globales (Gilardi, 2009). Los estudios comparados cualitativos subrayan relevantes diferencias y particularidades. La comparación cualitativa entre tradiciones de administración pública (Painter y Peters, 2010), o entre ARI (Thatcher, 2005) arroja luz sobre diferencias significativas. Para comparar dos países tan distintos como Dinamarca y España, sin una clara similitud institucional, es necesario un marco analítico más general y flexible. Se utiliza así una mayor abstracción y 
generalidad teórica en base a elementos comunes a la mayoría de las sociedades democráticas modernas, como puede ser la dimensión organizativa y pública.

\subsection{Altos técnicos o funcionarios en la administración pública}

Los altos técnicos en la administración pública de un estado representan la aspiración del modelo de conducta burocrática organizativa, al menos en la parte positiva del fenómeno. Para du Gay (2005), la forma organizativa burocrática significa la mejor contribución de la racionalidad organizativa: el comportamiento no sectario de una persona en sus decisiones que afectan a otras personas. En general, toda sociedad o estado que aspire a ser efectivo en la gestión de los asuntos públicos y generar prosperidad colectiva debe desarrollar una administración pública basada en la organización burocrática (Evans y Rauch, 1999). Painter y Peters (2010) subrayan diferencias significativas en las tradiciones de estado y de administración pública. En la tradición escandinava, el estado y el parlamento representan a una comunidad organizada, y la administración pública se caracteriza por un fuerte estatus profesional. En Dinamarca, en los ministerios los altos técnicos públicos tienen una alta autonomía y responsabilidad directamente por debajo del ministro-a; y los pocos cuerpos de funcionarios del estado que se desarrollaron ya apenas existen. En la tradición napoleónica o del sur de Europa el estado se concibe como un ente intervencionista sobre una comunidad de individuos, y el gobierno goza de un amplio margen de autonomía y poder. En España hay además una tradición de politización de los niveles altos de la administración pública (Sotiropoulos, 2004); por ejemplo, la figura de secretariado de estado quedó institucionalizada en 1976-78 como un cargo político con importantes funciones directamente por debajo del ministro-a.

\subsection{Participación en gobiernos: expertos académicos y partidos políticos}

La forma más directa de participación de economistas académicos es como ministro-a o en un cargo de alta responsabilidad ministerial. Esta forma de influencia conlleva un claro dilema entre el estatus científico y la práctica política respecto al partido político en el gobierno con el que se suele tener una vinculación organizativa o personal. La participación de políticos con estudios superiores en cargos de alta responsabilidad pública ha aumentado en las últimas décadas. Tavares de Almeida et al. (2004) observan en España, Grecia, Italia y Portugal una tendencia histórica al crecimiento de los perfiles técnicos en la política y en los gobiernos en detrimento de los perfiles solamente políticos, lo cual coincide con el aumento del nivel de satisfacción subjetiva de la ciudadanía con la política y la democracia. Hallerberg y Wehner (2013) recogen datos sobre la formación académica y profesional de 1200 responsables en política económica de los países de la OCDE en el periodo de 1973-2010, y presentan varias conclusiones: a) en los países de la zona Euro los responsables de política económica no suelen tener una formación en economía; b) los países con jóvenes democracias y con sistemas presidencialistas suelen tener ministro/as de economía y/o hacienda pública con formación técnica; y c) parece haber un patrón mayoritario de división del trabajo en los tres puestos principales: presidente o líder 
del gobierno sin formación en economía, la dirección del banco central con una alta formación académica, y ministro/a de economía y/o hacienda pública con o sin formación académica en economía.

Organizativamente, los políticos más o menos independientes y de perfil técnico suelen tener algún tipo de vinculación con el partido político en el gobierno. Los partidos políticos han ido aumentando la presencia de personas de perfil técnico, tanto dentro de sus órganos de decisión como en puestos de responsabilidad de gobierno. Esto puede entenderse dentro del proceso de racionalización de los partidos políticos para hacer frente a las complejidades de los subsistemas o sectores de la sociedad moderna, y para acercarse a los movimientos sociales y a la ciudadanía en general. Esto es a su vez parte del proceso de racionalización de las organizaciones civiles (Papakostas, 2011).

\subsection{Participación en organizaciones tecno-racionalizadas}

Hay dos tipos principales de organizaciones tecno-racionalizadas en política económica: los consejos asesores, y las agencias regulatorias independientes (ARI).

Órganos consultivos y de asesoramiento se han creado en algunos países, como Países Bajos (Centraal Planbureau CBP-Bureau for Economic Policy Analysis, creado en 1945), EUA (American Council of Economic Advisers, creado en 1946), Alemania (Sachverständigenrat zur Begutachtung der gesamtwirtschaftlichen Entwicklung - German Council of Economic Experts, creado en 1963), Dinamarca (De Økonomiske Råd - The Economic Council, creado en 1969), y el caso más reciente en Francia (Conseil d'analyse économique-CAE, en 1997). Los consejos de EUA y de Francia asesoran al presidente, y los miembros suelen cambiar cuando cambia el partido político en el gobierno. Los consejos de los Países Bajos, Alemania y Dinamarca asesoran al gobierno y al parlamento, y los miembros no suelen cambiar con el cambio de partido(s) político(s) en el gobierno. Davies (2011) analiza el surgimiento e institucionalización de esta figura de científico como asesor político a través de un estatus flexible.

Los economistas también participan en los bancos centrales y en las diversas ARI de la actividad económica. La creación e institucionalización de bancos centrales independientes se ha extendido a nivel global (Polillo y Guillén, 2005). Según Elgie (1998), en Europa desde finales de los años 80 la tendencia fue enfatizar las normas de independencia de los bancos centrales y de un menor control del gobierno, una tendencia promovida por la arquitectura del banco central de la Unión Europea (UE), a su vez inspirado en el banco central alemán. Sin embargo, las prácticas reales indican límites y problemas para una clara independencia. En la UE y en la zona Euro los bancos centrales han tenido un proceso de isomorfismo institucional, siguiendo a grosso modo la ideología ordoliberal, dominante en el pensamiento económico de Alemania. Según Dyson y Marcussen (2009), en la UE, en la era del Euro, el poder de los bancos centrales es menos visible que antes, ya que ahora se centran en la agenda de interpretación de los problemas y los intereses de la política ma- 
croeconómica en relación a cuestiones como competitividad, estabilidad y disciplina fiscal. Marcussen (2009) problematiza el cambio de racionalidad en los bancos centrales, y arguye que se ha pasado de discutir su autonomía o independencia a discernir su vinculación y nivel de cientificidad.

Las ARI han sido objeto de un número creciente de estudios. Su calificación de independiente asume que estas organizaciones deben aspirar a un alto nivel de autonomía, asumiendo que a mayor nivel de independencia técnica mayor nivel de racionalidad y eficacia en las decisiones reguladoras. Sin embargo, la investigación encuentra contradicciones y una difícil independencia. Fernández-i-Marin, et al. (2016) miden la independencia real o de hecho de las agencias regulatorias de España, analizando los rasgos, perfiles y tiempo de duración de 448 nombramientos de 325 personas en 12 agencias regulatorias entre 1979 y 2010, y encuentran un alto nivel de politización de los miembros y de su lógica de nombramiento. Destaca la relativamente alta presencia de personas vinculadas a partidos políticos y funcionarios con afinidades políticas, y hay un menor peso de personas de claro perfil técnico, especialmente aquellos con nivel educativo de doctorado. Asimismo, encuentran también interesantes diferencias entre las agencias cuyos miembros son nombrados por el gobierno y los nombrados por el parlamento. Estos datos coinciden con las tendencias de las ARI en los países europeos, al menos en la Europa continental. GarcíaJuanatey, et al. (2017) recogen las opiniones de los responsables de 137 agencias sobre su autonomía y la creciente rendición de cuentas ante el ministerio correspondiente, e interpretan las opiniones favorables a la rendición de cuentas de manera positiva como un avance en la calidad de la agentificación del estado en España.

Estos análisis adolecen de cierta asunción del imperativo tecnocrático. Presuponen la independencia técnica de estas organizaciones como fuente de una mayor efectividad y racionalidad pública, e indirectamente connotan que la lógica democrática no es una fuente para un adecuado equilibrio de la racionalidad pública. La independencia de los bancos centrales y las ARI supone un dilema entre la autonomía de la racionalidad técnica y el déficit democrático que conlleva la delegación de la política económica a un grupo de tecnócratas (Christensen y Lægreid, 2007). Es necesario enfatizar el diferente significado del papel de los parlamentos, gobiernos y ministerios en el control y supervisión de las ARI.

\section{ECONOMISTAS Y ORGANIZACIONES TECNO-RACIONALIZADAS EN LAS POLÍTICAS ECONÓMICAS DE ESPAÑA Y DINAMARCA}

\subsection{Tecnocracia público-administrativa en lo económico}

En España los altos cargos funcionariales o de nivel A han tenido y tienen un importante papel en el funcionamiento del estado (Villoria, 1999). Los economistas y abogados del es- 
tado y los inspectores de hacienda suelen ocupar responsabilidades importantes tanto en los ministerios como en las agencias reguladoras. En 1984 los economistas y los técnicos comerciales del estado se integraron en un mismo cuerpo. En este cuerpo llama la atención la desarrollada práctica de excedencia para trabajar en el sector privado. En un breve informe en el $2005^{4}$ la asociación de economistas del estado afirmaba: "En la actualidad, el Cuerpo está formado por un total de 560 miembros de los que 90 disfrutan de su jubilación y 470 trabajan. De estos últimos, 303 están activos en la Administración General del Estado, 36 están en régimen de servicios especiales y 131 trabajan en el sector privado en excedencia.» Es decir, un $27 \%$ estaba asesorando a empresas, lo que es un caso potencial de incompatibilidad y conflicto entre el interés general del estado y el de las empresas privadas. En los organigramas de los ministerios de hacienda y economía, ${ }^{5}$ se observa que debajo del nivel de ministro-a hay un nivel de secretarías y subsecretarías de perfil político, seguidos de cargos ocupados por altos técnicos del estado. ${ }^{6}$

En Dinamarca, los altos técnicos en los ministerios de temas económicos son profesionales sin estatus de funcionario, y vinculados a los estudios que organiza el sindicato DJøF (asociación de graduados en economía, derecho y ciencias político-sociales). Según Knudsen (2000), históricamente los altos técnicos del estado danés (embesdmcen) han gozado de un relativo espacio de poder y autonomía, dentro del control y legitimación del parlamento. No hay un cuerpo de funcionarios específico de economistas o técnicos comerciales, homologable al caso de España. En lo que respecta a la defensa legal de los intereses económicos del estado, ese servicio se hace a través de un bufete privado con un acuerdo-contrato de colaboración, dentro de la tradición del «kammeradvokat» (abogacía de cámara o cameral) que se remonta al siglo XVIII. En los organigramas de los ministerios de hacienda y de economía se observa que directamente debajo del ministro-a se sitúan técnicos públicos. ${ }^{7}$

En resumen, en España los niveles altos de la administración pública operan en la tradición de un control político por parte del gobierno; en Dinamarca los altos técnicos públicos tienen una tradición de independencia profesional respecto a la política.

\subsection{Economistas académicos en gobiernos y ministerios: ¿científicos o políticos?}

En España, en las últimas décadas ha aumentado la presencia de economistas académicos o con vinculación universitaria en los gobiernos tanto centrales como autonómicos. Según datos de Rodríguez-Teruel y Blondel (2011), de los 167 ministro/as entre los años 19772005, 100 tenían estudios universitarios (60 de Licenciatura y 40 de Master), por áreas (60 Derecho, 25 Ciencias Económicas, y 15 en las áreas científicas o técnicas); 41 eran altos

\footnotetext{
4 «75 años de técnicos comerciales y economistas del Estado», Revista Información Comercial Española, noviembre 2005. N. ${ }^{\circ} 826,7-9$.

${ }^{5}$ http://www.minhafp.gob.es (Hacienda) y http://www.mineco.es (Economía) (accedidos por última vez en diciembre 2017).

${ }^{6}$ Sobre los abogados del estado se puede consultar: https://www.ahorasemanal.es/un-cuerpo-de-elite-enexcedencia (accedido el 24 de abril 2017).

${ }^{7}$ www.fm.es (Hacienda) / www.oim.dk (Economía) (accedidos por última vez en diciembre 2017).
} 
funcionarios de la administración pública central, y 24 eran o habían sido académicos o profesores universitarios. En los altos cargos ministeriales de las áreas de economía, industria y hacienda destaca la creciente presencia de académicos o políticos con vinculación universitaria. Esta tendencia ha sido más marcada en los gobiernos del PSOE (19821996 y 2004-2011), con los casos de Solchaga, Croissier, Sebastián y Borrell, y a nivel de secretariado de estado (Trullén, Campa y Vegara); varios ministros y altos cargos eran economistas del estado (Solbes, Fernández-Ordoñez, de la Dehesa). En los gobiernos del PP (1996-2004 y 2011-2016) ha habido una tradicional presencia de políticos economistas del mundo privado (Piqué, Birulés, Rodrigo-Rato) y de economistas del estado como ministros (Guindos, Soria) y como secretarios de estado (Jiménez Latorre, los hermanos Nadal); y el caso de Montoro, catedrático de hacienda por la Universidad de Cantabria, y ministro en varias etapas. En los gobiernos de las Comunidades Autónomas también ha habido economistas académicos. En Cataluña los consejeros de economía entre 2003 y 2016 fueron catedráticos de economía (Castells 2003-2010 por el PSC, y Mas-Colell 2010-2016 por CIU). Casos parecidos se han dado en la Comunidad Valenciana con Soler y en Aragón con Bandrés, ambos del PSOE.

En Dinamarca en las últimas décadas no ha habido ningún economista académico como ministro/a o alto cargo ministerial. La ley de función pública tampoco ayuda de forma clara a ese tipo de excedencia. En este sentido Dinamarca es un caso complejo que requiere indagar en el funcionamiento del resto de las organizaciones. En concreto, en los partidos políticos daneses tiende a predominar la lógica democrática frente a la lógica técnica. Sin embargo, en la composición del parlamento, en 2015 el 62\% de los parlamentarios había cursado estudios relacionados con la asociación DJøF. ${ }^{8}$ Asimismo, en su estudio sobre la elite de poder en base a las diferentes relaciones organizativas en torno al estado (consejos, comisiones, órganos diversos, etc.), Ellersgaard, et al (2015) encuentran una importante presencia de catedráticos de economía ocupando puestos relevantes y compartiendo poder junto a directivos de grandes empresas y algunos líderes de sindicatos sectoriales.

Según estos datos, en los gobiernos de España ha habido una importante tendencia hacia una tecnocracia de economistas académicos-políticos; en los gobiernos de Dinamarca los ámbitos político y académico se han mantenido separados, pero con muchas instancias de relación, como es el caso del Consejo Económico, que se verá a continuación.

\subsection{Consejos asesores y ARI: organizaciones tecno-racionalizadas}

\subsection{1. Órganos o consejos asesores}

En España no existe un órgano independiente para asesorar en políticas económicas. Se pueden mencionar varias organizaciones que de alguna manera han hecho esa función:

\footnotetext{
${ }^{8}$ www.ft.dk (Parlamento de Dinamarca) (accedido por última vez en diciembre 2017).
} 
a) el Consejo Económico y Social, de composición tripartita, centrado en cuestiones sociolaborales; b) el Servicio de Estudios del Banco de España y la Oficina Económica vinculada a la Presidencia del gobierno han sido quizá los dos órganos donde la lógica economicista dominante ha tenido mayor recorrido e influencia en las políticas económicas; y c) el Consejo Empresarial de la Competitividad, creado en 2011, como reacción del gobierno del PSOE a la crisis económica, y eliminado en 2017, compuesto por las principales empresas del Ibex-35 y liderado por Telefónica y Banco Santander.

En Dinamarca el consejo asesor más relevante es el Consejo Económico (DøR), ${ }^{9}$ compuesto por: a) una presidencia científica de cuatro académicos (preferentemente catedrático-as) elegidos internamente dentro de la comunidad académica; y b) un pleno de 17 personas nombradas por el parlamento que representa los principales intereses políticos y económicos del país. La presidencia científica, desde una tradición de keynesianismo flexible, pragmático y moderado, realiza dos informes anuales, uno estructural y otro coyuntural, que son sometidos a debate en el pleno del consejo, cuyos comentarios se hacen públicos. DØR es relativamente costoso; su presupuesto equivale al programa estatal de investigación independiente. En su historia hay casos de influencia general, como en el referéndum sobre el Euro en el 2000: ante la opinión casi unánime de los partidos políticos y los sondeos a favor del ingreso en el Euro, la presidencia de DØR una semana antes opinó que desde la ciencia económica no había argumentos ni a favor ni en contra de la adhesión al Euro; el resultado final fue un 53,2\% en contra.

\subsubsection{Bancos Centrales}

El Banco de España (BdE), ${ }^{10}$ creado en 1782 como Banco San Carlos, fue regulado en 1980 en su versión actual. En 1993 se aprobó la Ley de Autonomía del BdE que establecía las normas destinadas a garantizar su autonomía e independencia. El gobernador/a del BdE es elegido por el gobierno, el cual elige al subgobernador/a, y al resto de los miembros del consejo del BdE y de la comisión ejecutiva. El consejo del BdE se compone de: a) 10 miembros con voto: gobernador/a y sub-gobernador/a, seis consejeros elegidos para un periodo de seis años (estos tres cargos deben tener un carácter técnico e independiente, con conocimiento y experiencia en el sector bancario y financiero), y la vice-dirección del órgano regulador del mercado financiero, y la secretaría del gobierno de política financiera; dos de los seis consejeros son elegidos por el gobernador para formar parte de la comisión ejecutiva; y b) 7 miembros sin voto: cinco directores del banco, un representante del personal y el secretariado del banco. En la historia reciente del BdE se pueden hacer dos observaciones. Primero, que los seis consejeros elegidos por el gobernador del banco han solido ser catedráticos de economía o técnicos en áreas relevantes; sin embargo, en el caso de los seis consejeros actuales, en base a sus breves CV disponibles en la página-web, no es fácil observar una clara independencia política. Segundo, para el puesto de gobernador

\footnotetext{
${ }^{9} \mathrm{http}: / /$ www.dors.dk (accedido por última vez en diciembre 2017).

${ }^{10} \mathrm{https} / / /$ www.bde.es (accedido por última vez en diciembre 2017).
} 
hay una tendencia a priorizar a los economistas del estado: de los seis gobernadores del BdE (Álvarez Rendueles (1978-1984), M. Rubio (1984-1992), A. Rojo (1992-2000), J. Caruana (2000-2006), M.A. Fernández-Ordoñez (2006-2012), L. M. Linde (2012-2018)), todos menos Rubio eran economistas funcionarios del estado; los tres primeros fueron también académicos universitarios; desde mayo del 2018 el gobernador es P. Hernández de Cos, con un perfil de macroeconomista clásico. En los últimos años el BdE ha recibido críticas: a) la supervisión del sector bancario antes de 2008 ha sido objeto de debate, polémica e incluso investigación judicial; ;1 b) en 2006 la asociación de inspectores del BdE denunció la pasividad y falta de control en la actuación del gobernador Caruana; ${ }^{12}$ y c) desde el 2008, bajo los mandatos de Fernández-Ordoñez y de Linde, por las decisiones de costosos rescates bancarios y de reestructuración del sistema bancario. ${ }^{13}$

En Dinamarca, el banco nacional o central ${ }^{14}$ fue creado en su versión actual en 1818, y en la reforma de 1936 quedó explícito su carácter independiente y autónomo en materia de política monetaria. El banco central danés tiene un organigrama a tres niveles: a) el consejo de representantes, compuesto por 25 miembros, que representa un amplio abanico de partes e intereses (ocho son miembros del parlamento, dos nombrados por el ministerio de industria-economía, dos son catedrático-as de universidad, y el resto es una representación de los diversos grupos de intereses, incluidos uno o dos representantes de los sindicatos); b) este consejo de representantes elige un consejo de administración, de siete miembros: dos nombrados por el ministerio de industria-economía, dos por el parlamento, y el resto en representación del consejo de representantes; y c) el consejo de administración elige la dirección del banco, compuesta por tres personas con experiencia en el sector financiero y bancario, y uno de ellos debe ser también de designación real. Según el $\mathrm{CV}$ de los tres directores actuales en la página-web, el director de designación real ha trabajado en el sector de las pensiones ${ }^{15}$ y los otros directores tienen un claro perfil técnico, con puestos previos en los ministerios de economía y hacienda y el banco central. En el

\footnotetext{
11 Sobre el caso de Bankia (antigua Caja Madrid), cuyo rescate público costó 22.242 millones de Euros, Climent y Pavia (2015) escriben: «En resumen, los problemas detectados podrían explicarse por alguno (o una combinación) de los siguientes motivos: (i) ocultación de pérdidas de préstamos en mora; (ii) no actualización de los activos al precio de mercado; o, (iii) exceso de provisiones. Aunque cada una de las posibles explicaciones supondría un grave problema de comportamiento y sería moralmente reprobable (sino legalmente punible), uno de los mayores problemas a nuestro juicio reside en que el ejercicio en que se acumularon la mayor parte de pérdidas no declaradas sirvió de información para realizar una OPS y una ampliación de capital en la que se obtuvo una financiación de más de 4.000 millones de euros (3.092,1 millones en la OPS del 15/7/11 y 1.155,0 millones en la ampliación de capital por conversión voluntaria de preferentes del 2/4/12), que en este momento no tienen ningún valor. Todo ello con el respaldo del Consejo de Administración de la entidad, de la empresa auditora Deloitte, del Banco de España, del Ministerio de Economía español, de la CNMV, de la Comisión Europea y de la EBA» (p. 22).

12 Se tiene acceso a las cartas de las denuncias, así como a otros fallos de la ciencia económica general y en España en el blog de Juan Torres (catedrático de economía aplicada): http://www.eldiario.es/temas/desvelando_ mentiras_mitos_y_medias_verdades_economicas/ (accedido por última vez en octubre 2017).

13 Según un informe del propio BdE de 2017, con datos y estimaciones a 31 de diciembre del 2015, de los 77.000 millones de Euros usados por el gobierno en los rescates bancarios se habían recuperado unos 4.000 y en el futuro se podrían quizá recuperar unos 12.000: https://www.bde.es/bde/es/secciones/informes/Otras_publicacio/informe-sobre-la/ (accedido el 12 de octubre de 2018).

${ }^{14}$ https://nationalbanken.dk (accedido por última vez en diciembre 2017).

15 En el complejo sistema de pensiones de Dinamarca hay dos principales tipos de pensiones que afectan a la mayoría de los ciudadanos: la pensión básica y general del estado, y las pensiones ocupacionales gestionadas por los diferentes sindicatos profesionales.
} 
otoño de 2008, para la gestión de la crisis financiera, el banco central y el gobierno: dispusieron una garantía bancaria ilimitada a un coste de 13.000 millones de Euros a pagar por los bancos, regularon la opción de intervenir bancos en crisis y la posibilidad de optar a ventajosos créditos estatales que se fueron formalizando hasta el otoño del 2013 a lo largo de cinco paquetes progresivos de medidas; en el 2016 un informe oficial afirmaba que el estado además de recuperar todo el dinero prestado había obtenido un beneficio de 2.400 millones Euros. ${ }^{16}$

Comparando los dos bancos centrales, en el caso español, cuya dirección es elegida por el gobierno, es difícil discernir entre lo técnico y lo político en los miembros con voto en el consejo del banco, mientras que en Dinamarca destaca el consenso parlamentario pluralista como base de legitimación y representación de las diferentes visiones e intereses.

\subsubsection{Agencias reguladoras del mercado financiero}

En España, la Comisión Nacional del Mercado de Valores (CNMV) ${ }^{17}$ es dirigida por un consejo compuesto por ocho miembros: presidencia, vicepresidencia, y seis consejeros de los que dos son fijos (secretaría de política financiera y subgobernador del BdE); cuatro consejeros forman el comité ejecutivo junto a los dos cargos presidenciales. En las presidencias de la CNMV desde 1988 (L. C. Croissier (1988-1996), J. Fernández-Armesto (1996-2000), P. Valiente (2000-2001), B. Calzada (2001-2004), M. Conthe (2004-2007), J. Segura (20072012), E. Rodríguez (2012-2016), y S. Albella (desde 2016)) predomina el perfil de tecnócrata público-político vinculado al partido gobernante, con formación económica en algunos casos. En el consejo de la CNMV hay una alta presencia de técnicos y abogados del estado. A fecha de noviembre de 2017, según los CV de los dos cargos presidenciales y de los seis consejeros disponibles en la página-web, se observa que el presidente, la vicepresidenta y los consejeros son mayormente economistas y abogados del estado con relación con el partido gobernante y en algunos casos con el sector privado.

El agente regulador de Dinamarca es el órgano de inspección financiera (Finanstilsynet). ${ }^{18}$ La comisión directiva de este órgano la componen siete miembros nombrados por el ministerio correspondiente. En 2014, con gobierno de centroizquierda, la comisión estaba compuesta por seis personas: dos académicos, dos personas con experiencia de gestión en el sector financiero, una con experiencia de gestión del sector empresarial en general, una en representación del banco central nacional, y el vicedirector del banco central de Suecia. Desde el 2016, con gobierno liberal-conservador, un puesto de académico ha sido sustituido por una persona de perfil de gestor de empresa privada. A mediados del 2018 unos periodistas de investigación han denunciado uno de los mayores casos de lavado de dinero

\footnotetext{
${ }^{16}$ La crisis bancaria fue objeto de un estudio por un equipo de más de 15 técnicos y altos cargos del estado, dirigido por cuatro catedráticos, hecho público en septiembre de 2013: https://www.dr.dk/nyheder/htm/Rangvid. pdf (accedido el 12 de octubre de 2018).

${ }^{17}$ https://www.cnmv.es (accedido por última vez en diciembre 2017).

${ }^{18}$ https://www.finanstilsynet.dk (accedido por última vez en diciembre 2017).
} 
en Europa, realizado por el mayor banco del país, Danske Bank, entre 2007 y 2015, a través de su filial en Estonia. En este caso, esta ARI ha sido criticada por una excesiva confianza hacia los datos, informaciones y análisis ofrecidos regularmente por el mismo banco. ${ }^{19}$

En suma, en España predomina el perfil de tecnócrata público-político con formación jurídica (abogados del estado). En Dinamarca se da un cierto equilibrio entre gestores privados, académicos y dos directores de bancos nacionales, pero este equilibrio y pluralismo en su composición no ha podido evitar fallos de control como el indicado.

\subsubsection{Agencia reguladora de la competencia}

En España la actual Comisión Nacional de Mercados y de la Competencia (CNMC) ${ }^{20}$ se creó a principios del 2013 integrando varias comisiones de competencia sectorial. Su composición de septiembre del 2013 fue criticada por no tener una clara competencia profesional. ${ }^{21}$ Desde su creación ha estado envuelta en polémicas sobre ceses y nombramientos, e incluso ha sido objeto de vigilancia por la Comisión Europea. ${ }^{22}$ De sus 10 miembros tres son académicos en economía (dos catedrático-as y un profesor-a titular), tres son técnicos del estado, dos han sido políticos, uno representante del sector de multimedia, y una persona con un perfil jurídico. El presidente desde 2013, Martin Quemada, tiene un cierto perfil académico con especialización en política energética.

En Dinamarca, el Consejo o Comisión de la Competencia en 2014 estaba compuesto por 18 miembros, de los que siete eran académicos (seis catedrático-as y un profesor-a titular), y en el resto había un equilibrio entre técnicos del estado, personas con experiencia del sector económico e intereses colectivos (tres miembros eran nombrados por los sindicatos). En diciembre del 2014 el parlamento aprobó una nueva ley de competencia que incluía un nuevo tipo de consejo de la competencia, pasando de una lógica de representatividad mixta técnico-política a una lógica claramente técnica, con un tamaño más reducido y especializado. ${ }^{23}$ El nuevo consejo se compone de siete miembros: cuatro deben tener conocimientos en derecho y economía, dos con experiencia de alta responsabilidad en la gestión privada y uno con conocimiento y experiencia en cuestiones de consumo. Este nuevo consejo de competencia adquiere la potestad de decidir las cuestiones o sectores a investigar sin el control del ministerio correspondiente. El ministerio puede también encargar estudios técnicos. En los informes se debe indicar si el análisis ha sido encargado por el consejo de competencia o por el ministerio. De los siete miembros del nuevo consejo de competencia para el periodo 2015-2019 cinco son catedrático-as y dos gestores privados.

\footnotetext{
19 https://www.dr.dk/nyheder/tema/hvidvask-i-danske-bank (accedido el 20 de octubre de 2018).

20 https://www.cnmc.es (accedido por última vez en diciembre 2017).

${ }^{21}$ Fernández-Villanueva y Garicano (El País, 27 de marzo de 2013 «Arbitrariedad y Competencia. La lista de los consejeros nombrados nos dice que la CNMC no va a cumplir con su misión»).

${ }^{22}$ El País, 26 de diciembre del 2013 «Bruselas mantiene bajo vigilancia al nuevo regulador de la Competencia».

${ }^{23}$ http://www.kfst.dk (accedido por última vez en diciembre 2017).
} 
En Dinamarca se observa una tendencia hacia una lógica tecnocrática-científica con una creciente presencia de economistas académicos. En España el gobierno ejerce un fuerte control sobre el órgano regulador, el cual tiende a tener un bajo perfil técnico-académico.

\subsubsection{La autoridad fiscal independiente}

En 2011 la Comisión de la UE estipuló la creación en cada país miembro de un organismo para supervisar la economía pública, con atención a la deuda y el déficit público. De esta manera se extendía a toda la UE la tradición de países como los Países Bajos (Kopits, 2012). En algunos países esta función fue asignada a organismos públicos ya existentes, como en Dinamarca, Países Bajos y Alemania. En otros países como en España se crearon organismos nuevos. En España, la Autoridad Independiente de Responsabilidad Fiscal (AiREF) ${ }^{24}$ se creó en 2014. España fue uno de los últimos países de la UE en crear este organismo. En el decreto ley de creación el gobierno tiene la potestad de nombrar los miembros: «La AiREF estará adscrita al Ministerio de Hacienda y Administración Pública, tendrá un presidente, asistido de directores de división. Todos sus miembros serán nombrados por el Gobierno entre profesionales con un mínimo de 10 años de experiencia profesional en análisis económico, financiero y presupuestario del sector público». ${ }^{25} \mathrm{El}$ presidente de la AiREF propone a las personas candidatas a la dirección de sus áreas o divisiones. El actual presidente de la AiREF, J. L. Escrivá, nombrado a principios del 2014, tiene experiencia profesional en el Banco Central Europeo y en un banco privado español. Esta nueva institución reproduce las tradiciones de control de las organizaciones públicas tecno-racionalizadas: por el gobierno en España y por el parlamento en Dinamarca.

\section{ANÁLISIS Y CONCLUSIONES}

Desde una sociología comparada se han analizado los factores organizativos en la racionalización de las políticas económicas en Dinamarca y España, dentro de la era de globalización económica neoliberal y su correspondiente creciente poder del capital y empresarial. Se ha propuesto los siguientes conceptos: a) el necesario equilibrio entre racionalidad técnica y racionalidad democrática para analizar la política económica en democracia; y b) la organización tecno-racionalizada para analizar la lógica técnica en la política económica. El fenómeno se ha analizado comparado la presencia, participación e influencia de economistas académicos y profesionales en tres contextos organizativos.

Primero, como altos técnicos públicos se ha observado en el caso danés una mayor independencia, autonomía y transparencia, y en el caso español una mayor vinculación y dependencia política, dentro de las diferentes tradiciones estatal y de administración pública. Segundo, respecto a la participación individual en gobiernos, en Dinamarca hay

\footnotetext{
${ }^{24}$ www.airef.es (accedido varias veces entre 2015 y 2017).

25 Ministerio de Economía: www.mineco.es (accedido en marzo 2015).
} 
históricamente una baja presencia de economistas académicos en los ministerios, mientras que en España ha habido una tendencia a la participación de economistas académicos en responsabilidades ministeriales, aunque dentro de un alto control político del gobierno. Tercero, en lo que atañe a la participación en órganos de asesoramiento, bancos centrales y agencias de regulación se puede extraer dos conclusiones: a) en España hay una limitada presencia de economistas académicos frente a la alta presencia de altos técnicos del estado como economistas y abogados del estado, sin que esté claramente delimitado su perfil técnico e independiente respecto a intereses políticos y privados; en Dinamarca la presencia de economistas académicos ha ido creciendo en colaboración con los tecnócratas públicos; b) en España el gobierno ejecutivo controla estas organizaciones públicas; en Dinamarca estas organizaciones son controladas por el parlamento que en tres de los cuatro casos analizados elige el primer nivel del organigrama a modo de base de representación compuesto por las principales fuerzas políticas en el parlamento y una representación de los principales intereses de la sociedad civil económica general y sectorial.

Respecto al necesario equilibrio entre racionalidad político-democrática y racionalidad técnico-científica en la política económica, en España predomina un modelo político-tecnocrático en el que el gobierno ejecutivo controla desde sus intereses políticos la relación entre las dos racionalidades; en Dinamarca predomina un modelo democrático-científico en el que el parlamento organiza el control y la representación de las dos racionalidades. Comparando las economías de los dos países, se observa la mejor evolución general y sectorial de la economía danesa, y por lo tanto se puede concluir que en el caso de la política económica danesa la racionalidad político-democrática ha sido un virtuoso contrapeso a la racionalidad técnico-científica.

\section{REFERENCIAS BIBLIOGRÁFICAS}

Adler, P.S. (ed.) (2009) The Oxford Hanbook of sociology and organization studies. Classical foundations. Oxford: Oxford University Press. https://doi.org/10.1093/oxfordhb/9780199535231.001.0001

Ahedo, M. (2018) Ciencia económica, gobierno y sistema de debate en la política económica de Dinamarca y España. Revista de Investigaciones Políticas y Sociológicas, 17(1), pp. 1-18.

Ahedo, M. (2017). Dinamarca \& España. Una sociología comparada, histórica y pública. Valencia: Tirant Lo Blanc.

Ban, C. (2016). Ruling Ideas: How global neoliberalism goes local. Oxford: Oxford University Press, https://doi.org/10.1093/acprof:oso/9780190600389.001.0001 
Beck, U. (2004). Poder y contrapoder en la era global. La nueva economía política mundial. Barcelona: Planeta.

Bourdieu, P. (2014). Sobre el Estado: Cursos en el Collége de france (1989-1992). Madrid: Anagrama.

Brint, S. (1994). In an age of experts. The changing role of professionals in politics and public life. Princenton: Princenton University Press.

Campbell, J. L. (1998). Institutional analysis and the role of ideas in political economy. Theory and Society, 27 (3), pp. 377-409. https://doi.org/10.1023/a:1006871114987

Centeno, M. A. (1993). The new Leviathan: The dynamics and limits of technocracy. Theory and Society, 22(3), pp. 307-335. https://doi.org/10.1007/bf00993531

Christensen, T., y Lægreid, P. (eds.). (2006). Autonomy and regulation: Coping with agencies in the modern state. Londres: Edward Elgar Publishing.

Christensen, T., y Lægreid, P. (2007). Regulatory agencies. The challenges of balancing agency autonomy and political control. Governance, 20(3), pp. 499-520. https://doi. org/10.1111/j.1468-0491.2007.00368.x

Climent, S., y Pavía, J. M. (2015). BANKIA: ¿Para qué sirven los estados contables y los órganos de control? / BANKIA: Financial Statements and Supervisory Bodies, Are they Really Useful?. Estudios de Economía Aplicada, 33 (1), pp. 259-300.

Collins, H., y R. Evans (2002). The third wave of Science Studies. Studies of expertise and experience. Social Studies of Science, 32 (2), pp. 235-296. https://doi. org/10.1177/0306312702032002003

Davies, W. (2011). Economic advice as a vocation: symbioses of scientific and political authority. The British journal of sociology, 62(2), pp. 304-323. https://doi.org/10.1111/ j.1468-4446.2011.01366.x

De Økonomiske Råd (2012). Jubilceumskrift De Økonomiske Råd 1962-2012, Copenhague (Libro 50. Aniversario del Consejo Económico, 1962-2012).

du Gay, P. (ed.) (2005). The Values of Bureaucracy. Oxford: Oxford University Press.

Dyson, K., y Marcussen, M. (eds.). (2009). Central banks in the age of the euro: Europeanization, convergence, and power. Oxford: Oxford University Press.

Elgie, R. (1998). Democratic accountability and central bank independence: Historical and contemporary, national and European perspectives. West European Politics, 21(3), pp. 53-76. https://doi.org/10.1080/01402389808425257 
Ellersgaard, C., Larsen, A. G. y Bernsen, M. (2015). Magteliten, hvordan 423 danskere styrer landet (La elite de poder. Cómo 423 daneses gestionan el país). Copenhague: Politiken.

Evans, P., y Rauch, J. E. (1999). Bureaucracy and growth: A cross-national analysis of the effects of» Weberian» state structures on economic growth. American sociological review, 64 (5), pp. 748-765. https://doi.org/10.2307/2657374

Fernández-i-Marín, X., Jordana, J., y Bianculli, A. C. (2016). Are regulatory agencies independent in practice? Evidence from board members in Spain. Regulation \& Governance, 10(3), pp. 230-247. https://doi.org/10.1111/rego.12084

Fischer, F. (2009). Democracy and expertise: Reorienting policy inquiry. Oxford: Oxford University Press. https://doi.org/10.1093/acprof:oso/9780199282838.001.0001

Fourcade, M. (2006). The construction of a global profession: The transnationalization of economics. American journal of sociology, 112(1), pp. 145-194. https://doi. org/10.1086/502693

Fourcade, M. (2009). Economists and societies: Discipline and profession in the United States, Britain, and France, 1890s to 1990s. Princenton: Princeton University Press. https:// doi.org/10.1515/9781400833139

García-Juanatey, A., Jordana, J., Parrado, S., Pascual, L., Salvador, M., y Sancho, D. (2017). Las agencias públicas en España: percepciones sobre autonomía y rendición de cuentas. Revista Española de Ciencia Política, (43), pp. 61-82. https://doi.org/10.21308/ recp.43.03

Gilardi, F. (2009). Delegation in the regulatory state: independent regulatory agencies in Western Europe. Londres: Edward Elgar Publishing. https://doi. org/10.4337/9781848441361

Hallerberg, M., y Wehner, J. (2013). The technical competence of economic policy-makers in developed democracies. Disponible: https://papers.ssrn.com/sol3/papers. cfm?abstract_id=2191490 (consultado el 15 de mayo de 2017)

Hirschman, D., y Berman, E. P. (2014). Do economists make policies? On the political effects of economics. Socio-Economic Review 12 (4), pp. 779-811. https://doi. org/10.1093/ser/mwu017

Kelly, T. (2004). Unlocking the iron cage: public administration in the deliberative democratic theory of Jürgen Habermas. Administration \& Society, 36(1), 38-61. https://doi. org/10.1177/0095399703257268 
Knudsen, T. (2000). Regering og embedsmcend. Om magt og demokrati $i$ staten (Gobierno y funcionarios. Sobre el poder y la democracia en el estado) Århus: Systime.

Kopits, G. (eds.) (2012). Restoring Public Debt Sustainability: the Role of Independent Fiscal Institutions. Oxford: Oxford University Press. https://doi.org/10.1093/acprof: oso/9780199644476.001.0001

Luhmann, N. (1995). Social Systems. Stanford: Stanford University Press.

Marcussen, M. (2009). Scientization of central banking: The politics of a-politicization. En Dyson, K., y Marcussen, M. (eds.). Central banks in the age of the euro: Europeanization, convergence, and power (pp. 373-390). Oxford: Oxford University Press.

Marres, N. (2017). Digital sociology: The reinvention of social research. Londres: John Wiley \& Sons.

Meyer, J. W., y Scott, W. R. (1992). Organizational environments: Ritual and rationality. Londres: Sage.

Pedersen, O. K., y Campbell, J. L (2014). The national origins of policy ideas: Knowledge regimes in the United States, France, Germany, and Denmark. Princenton: Princeton University Press. https://doi.org/10.1515/9781400850365

Painter M., y Peters, B.G. (2010). The Analysis of Administrative Traditions. En Painter, M. y Peters, B.G. (eds.) Tradition and Public Administration (pp. 3-16). Basingstoke, UK: Palgrave Macmillan. https://doi.org/10.1057/9780230289635_1

Papakostas, A. (2011). The rationalization of civil society. Current Sociology, 59 (1), pp. 5-23. https://doi.org/10.1177/0011392110385967

Polillo, S., y Guillén M.F. (2005). Globalization pressures and the state: the worldwide spread of Central Bank Independence. American Journal of Sociology, 110 (6), pp. 1764-1802. https://doi.org/10.1086/428685

Rodríguez-Teruel, R., y Blondel, J. (2011). Los Ministros de la España democrática: reclutamiento político y carrera ministerial de Suárez a Zapatero (1976-2010). Madrid: Centro de Estudios Políticos y Constitucionales.

Rodrik, D. (2011) The Globalization Paradox. Why global markets, states and democracy cannot coexist. Oxford: Oxford University Press.

Scott, W. R. (2013). Institutions and organizations: Ideas, interests, and identities. Londres: Sage. 
Sotiropoulos, D.A. (2004). Southern European public bureaucracies in comparative perspective. West European Politics, 27(3), pp. 405-422. https://doi. org/10.1080/0140238042000228077

Tavares de Almeida, P., Bermeo N., y Pinto, A.C. (eds.) (2004). Who Governs Southern Europe?: Regime Change and Ministerial Recruitment, 1850-2000. Londres: Routledge.

Thatcher, M. (2005). The third force? Independent regulatory agencies and elected politicians in Europe. Governance, 18(3), pp. 347-373. https://doi.org/10.1111/j.14680491.2005.00280.x

Villoria, M. (1999). El papel de la burocracia en la transición y consolidación de la democracia española: primera aproximación. Revista española de ciencia política, 1(1), pp. 97-126.

Woll, C. (2016). Politics in the interest of capital: A not-so-organized combat. Politics \& Society, 44(3), pp. 373-391. https://doi.org/10.1177/0032329216655318 